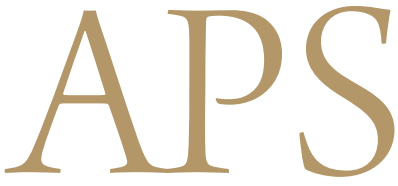

Archives of Plastic Surgery

\title{
Combined Orbital Fractures: Surgical Strategy of Sequential Repair
}

\author{
Su Won Hur, Sung Eun Kim, Kyu Jin Chung, Jun Ho Lee, Tae Gon Kim, Yong-Ha Kim \\ Department of Plastic and Reconstructive Surgery, Yeungnam University College of Medicine, Daegu, Korea
}

Background Reconstruction of combined orbital floor and medial wall fractures with a comminuted inferomedial strut (IMS) is challenging and requires careful practice. We present our surgical strategy and postoperative outcomes.

Methods We divided 74 patients who underwent the reconstruction of the orbital floor and medial wall concomitantly into a comminuted IMS group (41 patients) and non-comminuted IMS group (33 patients). In the comminuted IMS group, we first reconstructed the floor stably and then the medial wall by using separate implant pieces. In the non-comminuted IMS group, we reconstructed the floor and the medial wall with a single large implant.

Results In the follow-up of 6 to 65 months, most patients with diplopia improved in the firstweek except one, who eventually improved at 1 year. All patients with an EOM limitation improved during the first month of follow-up. Enophthalmos (displacement, $2 \mathrm{~mm}$ ) was observed in two patients. The orbit volume measured on the CT scans was statistically significantly restored in both groups. No complications related to the surgery were observed.

Conclusions We recommend the reconstruction of orbit walls in the comminuted IMS group by using the following surgical strategy: usage of multiple pieces of rigid implants instead of one large implant, sequential repair first of the floor and then of the medial wall, and a focus on the reconstruction of key areas. Our strategy of step-by-step reconstruction has the benefits of easy repair, less surgical trauma, and minimal stress to the surgeon.

Keywords: Orbital fractures / Fractures, comminuted / Facial bones
Correspondence: Yong-Ha Kim Department of Plastic and Reconstructive Surgery, Yeungnam University College of Medicine, 170 Hyeonchung-ro, Nam-gu, Daegu 705-717, Korea Tel: $+82-53-620-3483$ Fax: +82-53-626-0705 E-mail: kimyon@ynu.ac.kr

This article was supported by Yeungnam University grants in 2014.

This article was presented as a poster at the 2014 Congress of the European Association for Cranio-Maxillo-Facial Surgery in Prague, Czech Republic, on September 23-26, 2014.

No potential conflict of interest relevant to this article was reported.

Received: 15 Apr 2015• Revised: 1 Jun 2015• Accepted: 1 Jun 2015

pISSN: 2234-6163 • elSSN: 2234-6171 • http://dx.doi.org/10.5999/aps.2015.42.4.424• Arch Plast Surg 2015;42:424-430

\section{INTRODUCTION}

The spectrum of the severity of orbital wall fractures ranges from simple linear fractures to comminuted fractures. The two most common orbital fractures are floor and medial wall fractures. Surgery for combined orbital floor and medial wall fractures with a comminuted inferomedial strut (IMS) can be challenging; thus, taking special care is mandatory. Poor management in the initial operation can result in a catastrophe for each patient. Patients who had secondary enophthalmos may find it difficult to return to their normal social life because of serious functional and cosmetic sequelae, such as visual alteration, diplopia, and enophthalmos.

The goal of the initial surgery of combined orbital wall fractures is to reconstruct the bony orbital volume while maintaining a harmonious balance with soft tissue. To construct a proper orbit, the surgeon should rebuild the radius appropriately, including well-contoured orbital walls and a stable IMS, that is, the maxillo-ethmoidal buttress between the orbital floor and 
the orbital medial wall. The significance of IMS in the treatment of combined orbital floor and medial wall fractures can be considered a cornerstone over which implants can be placed stably [1-3].

In treating combined orbital fractures, the surgeon has two choices: the use of an implant with multiple small pieces or the use of a single large implant. When the orbit was reconstructed using the first method, the orbit could be rebuilt easily. However, there was a possibility of implant buckling and displacement if the materials were not rigid. Sometimes, using a third implant at the junction of the floor and the medial wall implants is necessary, for avoiding unstable restoration of the IMS. Reconstruction of the orbital walls by using the second method seems ideal and may result in excellent overall outcomes. However, wide exposure for the placement of a single large implant can lead to severe edema or hematoma postoperatively. Sometimes, impellent attempts such as by repeated insertion or repositioning of the implant may cause secondary trauma to the soft tissues and result in worsened or new-onset visual symptoms.

In this study, we suggest a surgical strategy to reconstruct the orbit by repairing the IMS, the floor, and the medial wall for patients with combined orbital floor and medial wall fractures. Here, we present our experience and evaluate the postoperative outcomes.

\section{METHODS}

From 2006 to 2014, a retrospective chart review was performed on 74 patients who underwent the reconstruction of the orbital floor and the medial wall concomitantly. Patients with pure or impure orbital wall fractures were included, and patients with bilateral fractures or small fractures were excluded. We divided the patients into the comminuted IMS group and the non-comminuted IMS group according to whether the IMS was comminuted on the preoperative computed tomography (CT) scan or not. Among them, 41 patients were included in the comminuted group and 33 patients in the non-comminuted group. The surgical indications were a defect size larger than $2 \mathrm{~cm}^{2}$, wall displacement of $\geq 3 \mathrm{~mm}$ on the CT scan, or other defects and displacement that may cause secondary enophthalmos. The patient age ranged from 17 to 72 years (mean, 34.9 years). There were 61 males and 13 females in the study population.

\section{Preoperative assessment}

Before surgery, all patients underwent accurate clinical evaluations for diplopia, extraocular muscle (EOM) limitation, any accompanying eye diseases, or any other symptoms. The presence of enophthalmos was determined with a Hertel exophthalmometer after the resolution of traumatic edema. The defect size of the orbital wall and the extent of wall displacement were measured from the facial CT scan by using the caliper function on the hospital's imaging system. Different operative plans were determined according to the comminuted extent of the orbital wall and the existence of compromised IMS on the facial CT scan.

\section{Operative technique}

All operations were performed by a senior surgeon after the resolution of traumatic edema. The average time of surgery was 10.3 days after trauma (range, 5 to 24 days).

Combined orbital floor and medial wall fractures with comminuted IMS (Fig. 1)

In cases of combined orbital floor and medial wall fractures with severely displaced IMS, we planned to reconstruct the floor and the medial wall separately: first reconstructing the floor stably and then reconstructing the medial wall.

For exploring the floor, we used transcutaneous or transconjunctival approaches. The orbital branch of the infraorbital artery was electrocauterized for preventing delayed postoperative hematoma, and the fine ligaments connecting between the infraorbital nerve and the periorbita were cut and dissected to obtain a wide surgical field. Then, the medial wall was accessed using the transcaruncular approach. The inferior and medial dissections were joined to create a single dissection plane and all prolapsed orbital soft tissues were restored.

By introducing the periosteal elevator into the maxillary sinus through the fractured hole, we detected the margins of the fracture area and measured the extent of the defect. The defect site on the floor was covered by a 0.4-mm-thick rigid preformed titanium implant (MatrixORBITAL, DePuySynthes Inc., West Chester, PA, USA).

Then, the tip of the elevator was inserted into the ethmoidal sinus and advanced toward the medial orbital wall, confirming the margins of the fracture area. The 3-mm-thick porous polyethylene sheets (SynPOR, DePuySynthes Inc.) was cut into pieces and inserted into the ethmoidal sinus as our previously reported inlay technique in order to compensate for the loss of medial support [4]. The previously inserted titanium mesh on the floor was used as the cornerstone for the optimal reconstruction of the medial wall.

Combined orbital floor and medial wall fractures with noncomminuted IMS (Fig. 2)

When the fractures were non-comminuted, IMS was discernible in the CT or operative field. In such cases, we tried to recon- 


\section{Fig. 1. Facial CT scans and photographs of case 1}

The combined orbital floor and medial wall fractures with comminuted inferomedial strut (IMS) was identified in the computed tomography (CT) scans. We constructed the IMS first by inserting the preformed titanium mesh on the orbital floor and subsequently, compensated for the loss of medial support with three pieces of a porous polyethylene sheet. The preformed titanium mesh was used as the cornerstone for the optimal reconstruction of the medial wall. (A) Preoperative CT scans (orbital volume, right $24.61 \mathrm{~cm}^{3}$; left $28.92 \mathrm{~cm}^{3}$ ), (B) immediately postoperative CT scans (red arrow, preformed titanium mesh; yellow arrow, porous polyethylene sheets) (orbital volume, right $24.61 \mathrm{~cm}^{3}$; left $\left.24.79 \mathrm{~cm}^{3}\right)(\mathrm{C}) \mathrm{frontal}$ view of the preoperative photograph (D) worm's eye view of the preoperative photograph (Hertel exophthalmometry, base $113 \mathrm{~mm}$; right $18 \mathrm{~mm}$, left $16 \mathrm{~mm}$ ) (E) frontal view of the postoperative 23-month follow-up photograph (F) worm's eye view of the postoperative 23-month follow-up photograph (Hertel exophthalmometry, base $113 \mathrm{~mm}$; right $18 \mathrm{~mm}$; left $18 \mathrm{~mm}$ ).
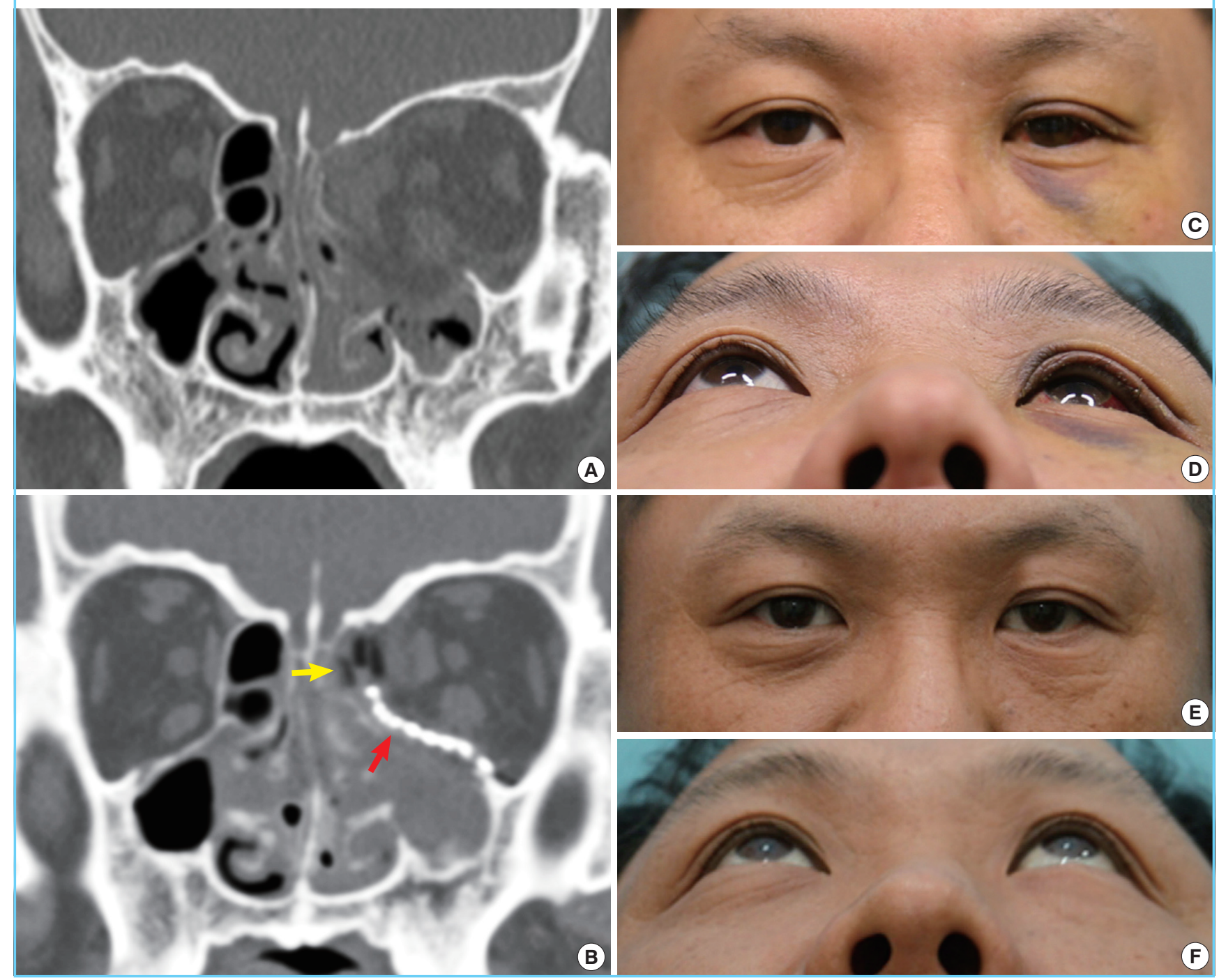

struct the floor and the medial wall with a single large preformed titanium implant. However, when IMS was compromised with instability intraoperatively despite appearing intact on the preoperative CT scan, or when the surgeon found it difficult to position the single large implant properly, the operative plan was changed to reconstruct the floor and the medial wall by using separate pieces of implants and sequentially repairing first the floor stably and then the medial wall. In cases of minor fractures, we tried to reconstruct only the displaced key area.

\section{Postoperative assessment}

Visual symptoms such as diplopia and EOM limitations were checked as soon as the patients were awakened from anesthesia, and a CT scan was performed immediately after the operation to confirm whether the implants were well-positioned or not. All of the patients were followed up at 1, 6, and 12 months postoperatively, and we checked for visual symptoms, presence of enophthalmos, and the occurrence of complications at these follow-ups. For preoperative and postoperative functional assessments, the level of diplopia and the range of ocular move- 


\section{Fig. 2. Facial CT scans and photographs of case 2}

The combined orbital floor and medial wall fractures with non-comminuted inferomedial strut (IMS) were identified in the computed tomography (CT) scans. Although the IMS looked stable in the CT scans, the IMS stability should be checked again intraoperatively. We reconstructed the floor and medial wall with a single large preformed titanium mesh. (A) Preoperative CT scans (orbital volume, right $24.06 \mathrm{~cm}^{3}$; left $\left.27.23 \mathrm{~cm}^{3}\right)(B)$ immediate postoperative CT scans (red arrow, preformed titanium mesh) (orbital volume, right $24.06 \mathrm{~cm}^{3}$; left $24.21 \mathrm{~cm}^{3}$ ) (C) frontal view of preoperative photograph (D) worm's eye view of the preoperative photograph (Hertel exophthalmometry, base $111 \mathrm{~mm}$; right $17 \mathrm{~mm}$; left $14 \mathrm{~mm}$ ) (E) frontal view of the postoperative 20-month follow-up photograph (F) worm's eye view of postoperative 23-month follow-up photograph (Hertel exophthalmometry, base $111 \mathrm{~mm}$; right $17 \mathrm{~mm}$; left $17 \mathrm{~mm}$ ).
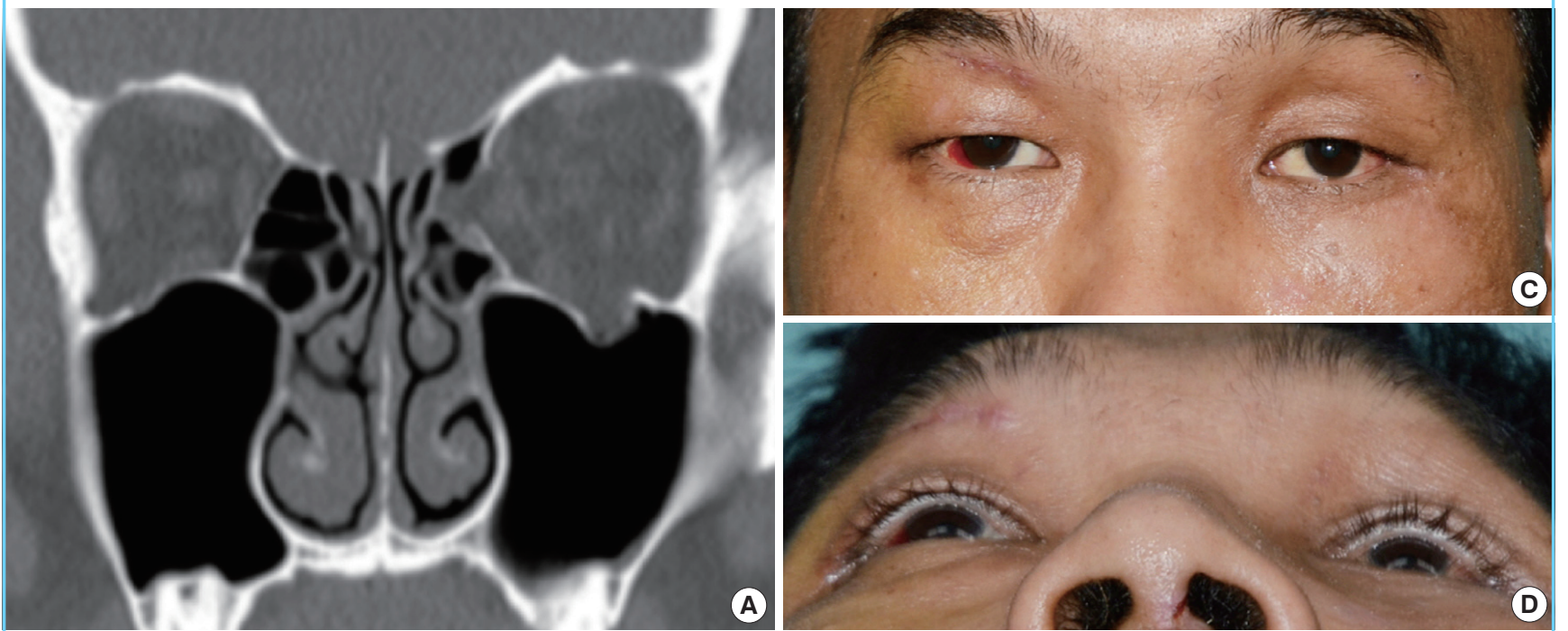

(A)
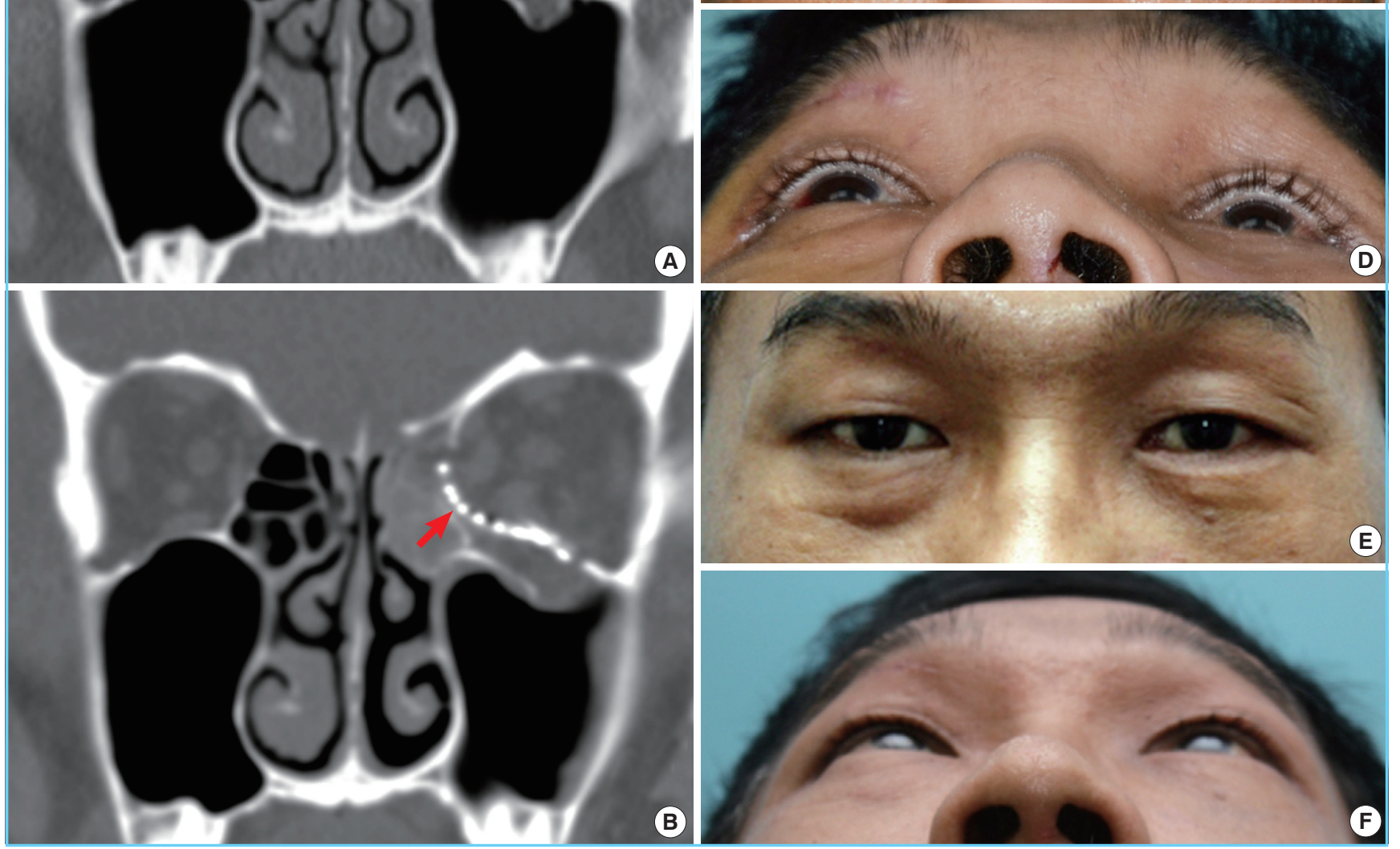

ment were compared. For an aesthetic assessment, the degree of correction and the extent of enophthalmos were measured, and the preoperative and postoperative CT scans were compared.

\section{Statistical analysis}

The preoperative and postoperative orbital volumes were measured on the CT scans by using Aquarius Workstation (iNtuition Aquarius, version 4.4.6; TeraRecon Inc., CA, USA) and analyzed statistically by using a paired $t$ test with SPSS 13.0 software (SPSS Inc., Chicago, IL, USA). A value of $\mathrm{P}<0.05$ indicated statistical significance.

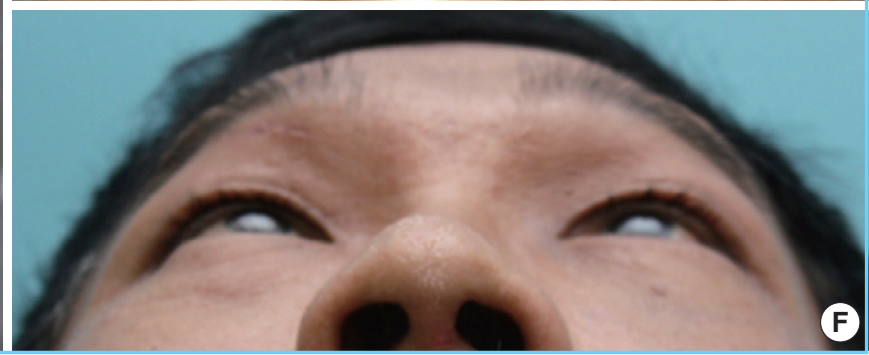

\section{RESULTS}

In the follow-up of 6 to 65 months (average, 34.1 months), one patient had persistent diplopia for more than 6 months, which eventually decreased at 1 year after surgery. Most patients presenting with diplopia improved in the first week. All patients with EOM limitations improved during the first month of follow-up. Those who had enophthalmos of more than $2 \mathrm{~mm}$ before surgery improved to the normal range after surgery. Enophthalmos of $2 \mathrm{~mm}$ was observed in two patients (Table 1 ).

The orbit volume was statistically significantly restored in both 
Table 1. Symptoms and presence of enophthalmos in combined orbital floor and medial wall fractures

\begin{tabular}{|c|c|c|c|c|c|c|c|c|c|c|}
\hline \multirow{3}{*}{ Symptoms } & \multicolumn{5}{|c|}{ Comminuted IMS group $(n=41)$} & \multicolumn{5}{|c|}{ Non-comminuted IMS group $(n=33)$} \\
\hline & \multirow{2}{*}{ Preoperative $^{\text {a) }}$} & \multicolumn{4}{|c|}{ Postoperative $^{\mathrm{b})}$} & \multirow{2}{*}{ Preoperative } & \multicolumn{4}{|c|}{ Postoperative } \\
\hline & & Immediate & $1 \mathrm{mo}$ & $6 \mathrm{mo}$ & $1 \mathrm{yr}$ & & Immediate & $1 \mathrm{mo}$ & $6 \mathrm{mo}$ & $1 \mathrm{yr}$ \\
\hline Diplopiac) & 22 & 16 & 7 & 1 & 0 & 17 & 10 & 3 & 0 & 0 \\
\hline EOM limitation & 9 & 6 & 0 & 0 & 0 & 7 & 2 & 0 & 0 & 0 \\
\hline Enophthalmos ${ }^{\text {e) }}(\geq 2 \mathrm{~mm})$ & 21 & - & 2 & 2 & 2 & 9 & - & 0 & 0 & 0 \\
\hline
\end{tabular}

IMS, inferomedial strut; EOM, extraocular muscle.

a)Preoperative symptoms and the presence of enophthalmos were checked after the resolution of traumatic edema, approximately 1 week after the trauma; blmmediate postoperative symptoms were checked as soon as the patients were awakened from anesthesia. All of the patients were followed up at 1, 6, and 12 months postoperatively, and

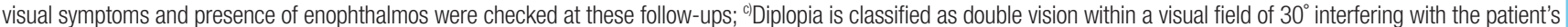
daily activities; 'Extraocular muscle limitations are determined by the forced duction test and assessment of double vision; ${ }^{\text {eT}}$ The corrected enophthalmos defined as the difference of Hertel exophthalmometry is less than $2 \mathrm{~mm}$ between both globes.

Table 2. Mean volume of non-fractured and fractured orbit in combined orbital floor and medial wall fractures

\begin{tabular}{|c|c|c|c|c|}
\hline \multirow{2}{*}{ Mean orbital volume } & \multicolumn{2}{|c|}{ Comminuted IMS group $(n=41)$} & \multicolumn{2}{|c|}{ Non-comminuted IMS group $(n=33)$} \\
\hline & Non-fractured orbit $\left(\mathrm{cm}^{3}\right)$ & Fractured orbit $\left(\mathrm{cm}^{3}\right)$ & Non-fractured orbit $\left(\mathrm{cm}^{3}\right)$ & Fractured orbit $\left(\mathrm{cm}^{3}\right)$ \\
\hline Preoperative & $24.65 \pm 2.24$ & $29.67 \pm 4.36$ & $24.31 \pm 3.06$ & $27.51 \pm 3.28$ \\
\hline Postoperative ${ }^{\text {a) }}$ & $24.65 \pm 2.24$ & $24.71 \pm 1.81$ & $24.31 \pm 3.06$ & $24.22 \pm 2.45$ \\
\hline P-value $e^{\text {b) }}$ & - & 0.006 & - & 0.013 \\
\hline
\end{tabular}

groups (Table 2). In the comminuted fracture group, the preoperative mean orbital volume was $29.67 \pm 4.36 \mathrm{~cm}^{3}$ and the postoperative volume was $24.71 \pm 1.81 \mathrm{~cm}^{3}(\mathrm{P}=0.006)$. In the noncomminuted fractured group, the preoperative volume was 27.51 $\pm 3.28 \mathrm{~cm}^{3}$ and the postoperative volume was $24.22 \pm 2.45 \mathrm{~cm}^{3}$ $(\mathrm{P}=0.013)$.

There were no complications such as infection, hemorrhage, or development of visual symptoms during the follow-up period. None of the implants showed any evidence of exposure or migration. Two patients complained of visual disturbances after awakening from anesthesia. Immediate additional procedures were undertaken to reposition the implants, and the symptoms were resolved.

\section{Case 1}

A 39-year-old man was admitted to the emergency room with blunt trauma on the left side of his face caused by a steel bar hitting him during industrial work. At the time of admission, diplopia was observed in the upper and lateral gaze. Hertel exophthalmometry revealed an anterior corneocanthal displacement of $18 \mathrm{~mm}$ in the left eye and $16 \mathrm{~mm}$ in the right eye with an interlateral canthal base of $113 \mathrm{~mm}$ after the resolution of traumatic edema. The facial CT scan showed combined orbital floor and medial wall fractures with severely displaced IMS on the left side. We restored the transition zone first with the fixation of the preformed titanium mesh on the orbital floor through a combined transcaruncular-subciliary approach. Subsequently, one 3-mm-thick porous polyethylene sheet was inserted to reconstruct the orbital medial wall over the just-inserted preformed titanium mesh, which was used as the cornerstone for the optimal reconstruction of the medial wall. The compromised IMS, orbital floor and medial wall were stably restored postoperatively. The fractured orbital volume before the surgery was 28.92 $\mathrm{cm}^{3}$ and that after the surgery was $24.79 \mathrm{~cm}^{3}$. Hertel exophthalmometry revealed an anterior corneocanthal displacement of $18 \mathrm{~mm}$ in the right eye, and the diplopia disappeared after the surgery. No complications were observed during the 23-month follow-up period (Fig. 1).

\section{Case 2}

A 35-year-old man was admitted to the emergency room with blunt trauma on the left side of his face caused by a beating. At the time of admission, diplopia was observed in the lateral gaze, and an EOM limitation was identified in the left lateral gaze. Hertel exophthalmometry revealed an anterior corneocanthal displacement of $17 \mathrm{~mm}$ in the left eye and $14 \mathrm{~mm}$ in the right eye with an interlateral canthal base of $111 \mathrm{~mm}$ after the resolution of traumatic edema. The facial CT scan showed combined orbital floor and medial wall fractures without displaced IMS on the left side. We trimmed the preformed titanium mesh so that 
the outer circumference was as small as possible but still provided sufficient width to cover the defect from the orbital floor to the orbital medial wall. The orbital floor and medial wall were properly restored with a single large implant postoperatively. The fractured orbital volume before surgery was $27.23 \mathrm{~cm}^{3}$ and that after surgery was $24.21 \mathrm{~cm}^{3}$. Hertel exophthalmometry revealed an anterior corneocanthal displacement of $17 \mathrm{~mm}$ in the right eye, and the EOM limitation and diplopia were resolved after surgery. No complications were observed during the 20-month follow-up period (Fig. 2).

\section{DISCUSSION}

Various techniques to deal with combined orbital floor and medial wall fractures have been described, and many surgeons have reported good results with their familiar approach and materials [4-10]. However, thus far, little attention has been paid to combined orbital floor and medial wall fractures with comminuted IMS.

Several researchers have reconstructed the orbital floor and medial wall concomitantly by using multiple small pieces of implants. Su and Harris [5] reported that they repaired combined orbital walls with overlapping two or three nylon foil implants (thickness, 0.2-0.4 mm) through combined transcarunculartransconjunctival incisions. They demonstrated the relative ease of insertion of the relatively small implants used in their technique. The limitations of their study were the small number of cases considered and suspected instability of the IMS area caused by the use of a non-rigid implant. Choi et al. [6] proposed a technique involving the use of two separate porous polyethylene channel implants (thickness, $2.3 \mathrm{~mm}$ ), fixated to the orbital rim by titanium plates placed within the longitudinal channels. The plates can be used to cantilever the implants into an anatomic position in the absence of an internal bony support. However, these implants are relatively thick and have the potential to cause postoperative hyperglobus.

On the other hand, some surgeons repaired the combined orbital wall fractures by using a single large implant. Nunery et al. [7] reported that they repaired combined orbital walls with a single large nylon foil implant (thickness, $0.4 \mathrm{~mm}$ ) by using a transcaruncular-transconjunctival approach. Although their overall results were good, their short-term follow-up (mean, 6.2 months) was not sufficient to prove the long-term stability of IMS. The excessive bowing of non-rigid nylon foil implant at the IMS could cause late enophthalmos. Several other authors described the usage of rigid implants such as porous polyethylene, polyethylene-embedded titanium mesh implants, or titanium mesh implants [8-10]. These techniques seem ideal theo- retically, if the implants are placed properly. However, their limitations include the wide exposure for the placement of the single large implant, resulting in severe edema or hematoma postoperatively. Furthermore, impellent attempts such as repeated insertion or repositioning of the implant could cause secondary trauma and lead to unexpected complications. Cho and Davies [8] reported a high revision rate (about 20\%) in their study and confessed the difficulty of inserting a single large implant.

Compared with previously reported studies, our study had more cases, a longer follow-up duration, and fewer complications. We could reconstruct IMS-comminuted orbit walls by using the following surgical strategy: usage of multiple pieces of rigid implants instead of one large implant, the sequential repair of first the floor stably and then the medial wall, and a focus on the reconstruction of the key areas of the orbit. Reconstructing orbital walls with separate pieces of implants and familiar approaches can reduce the risk of secondary surgical trauma. Sequential repair of first the floor by inserting the preformed titanium mesh provided the support for the optimal placement of the implant in the medial wall, and thus, the compromised IMS could be stably restored. The other advantages of this method are that the surgeon could insert the implant easily, position the implant more properly, and prevent severe edema or hematoma postoperatively because of the use of separate small incisions.

Focusing the reconstruction of orbital key areas instead of obsession to reconstruct all fractured sites completely could lead sufficient acceptable results. This simplified concept could save time and reduce surgery-related complications. The key areas of the orbit include the maxillo-ethmoidal buttress, inferomedial bony ledges, $\mathrm{S}$-shape of the orbital floor in the posterior third, and the inferior two-thirds of the medial wall with a bulge towards the orbit $[11,12]$. When shaping the implant prior to insertion, careful attention must be paid to replicating the slopes and curves of these key areas.

In particular, the IMS, or maxillo-ethmoidal buttress, is a critical structure as it provides appropriate orbital volume and determines the globe position. Unstable reconstruction of the compromised strut may lead to an undesirable secondary change of the orbital contour and consequently, lead to the development of enophthalmos or dystopia. To reconstruct the IMS stably in the combined orbital floor and medial wall fractures, thin and rigid implants are mandatory. The lack of malleability of the thick implants is problematic when attempting to replicate the normal contours of the floor and the medial wall. In contrast, a very flexible implant raises the concern of implant buckling and displacement. We believe that a $0.4-\mathrm{mm}$-thick preformed titanium mesh implant is suitable for the stable restoration of the compromised floor and IMS because of many advantages such as 
the minimal contouring, thin profile, ease of shaping anatomically, and stability over time $[8,9]$. For medial wall repair, we used a separate implant to avoid surgical trauma. Our method of inlay implantation, filling the ethmoidal sinus with several pieces of porous polyethylene, produced good outcomes from the anatomical reconstruction of the orbital wall and was free of surgery-related complications [4].

In this study, we analyzed the pros and cons of previously reported surgical procedures and added our experience and concepts of surgery. We suggest reconstructing first the floor stably and then the medial wall by using rigid implants with separate pieces and several approaches during the reconstruction of combined orbital floor and medial wall fractures with comminuted IMS. Our strategy of step-by-step reconstruction has advantages of easy repair, less surgery-related trauma, and minimal stress to the surgeon.

\section{REFERENCES}

1. Kim JW, Goldberg RA, Shorr N. The inferomedial orbital strut: an anatomic and radiographic study. Ophthal Plast Reconstr Surg 2002;18:355-64.

2. Wright ED, Davidson J, Codere F, et al. Endoscopic orbital decompression with preservation of an inferomedial bony strut: minimization of postoperative diplopia. J Otolaryngol 1999;28:252-6.

3. Burm JS, Chung CH, Oh SJ. Pure orbital blowout fracture: new concepts and importance of medial orbital blowout fracture. Plast Reconstr Surg 1999;103:1839-49.

4. Kim YH, Kim TG, Lee JH et al. Inlay implanting technique for the correction of medial orbital wall fracture. Plast Reconstr Surg 2011;127:321-6.
5. Su GW, Harris GJ. Combined inferior and medial surgical approaches and overlapping thin implants for orbital floor and medial wall fractures. Ophthal Plast Reconstr Surg 2006; 22:420-3.

6. Choi JC, Fleming JC, Aitken PA, et al. Porous polyethylene channel implants: a modified porous polyethylene sheet implant designed for repairs of large and complex orbital wall fractures. Ophthal Plast Reconstr Surg 1999;15: 56-66.

7. Nunery WR, Tao JP, Johl S. Nylon foil "wraparound” repair of combined orbital floor and medial wall fractures. Ophthal Plast Reconstr Surg 2008;24:271-5.

8. Cho RI, Davies BW. Combined orbital floor and medial wall fractures involving the inferomedial strut: repair technique and case series using preshaped porous polyethylene/titanium implants. Craniomaxillofac Trauma Reconstr 2013;6: 161-70.

9. Scolozzi P, Momjian A, Heuberger J, et al. Accuracy and predictability in use of $\mathrm{AO}$ three-dimensionally preformed titanium mesh plates for posttraumatic orbital reconstruction: a pilot study.J Craniofac Surg 2009;20:1108-13.

10. Lee KM, Park JU, Kwon ST, et al. Three-dimensional prebent titanium implant for concomitant orbital floor and medial wall fractures in an East asian population. Arch Plast Surg 2014;41:480-5.

11. Fan X, Li J, Zhu J, et al. Computer-assisted orbital volume measurement in the surgical correction of late enophthalmos caused by blow out fractures. Ophthal Plast Reconstr Surg 2003;19:207-11.

12. Kempster R, Beigi B, Galloway GD. Use of enophthalmic implants in the repair of orbital floor fractures. Orbit 2005; 24:219-25. 